Instituto Agronômico de Pernambuco (IPA), Av. Gal. San Martin, 1371, Bonji, CEP 50761-000, Recife, PE, Brasil

2Universidade Federal do Recôncavo da Bahia (UFRB), Cruz das Almas, BA, Brasil ${ }^{3}$ Embrapa Algodão, Campina Grande, PB, Brasil

*autor correspondente

凶servulo.siqueira@ipa.br

http://dx.doi.org/10.12661/pap.2014.010

\title{
Lâminas de irrigação e populações de plantas na mamoneira cV BRS Energia
}

\author{
Depths of irrigation and plant population in castor bean $\mathrm{CV}$ \\ BRS Energia
}

Sérvulo Mercier Siqueira e Silva ${ }^{*}$, Hans Raj Cheyi ${ }^{2}$, Napoleão Esberard de Macêdo Beltrão ${ }^{3}$

José Marcelo Dias ${ }^{3}$, Liv Soares Severino ${ }^{3}$, José Wellingthon dos Santos ${ }^{3}$, Farnésio de Sousa Cavalcante

RESUMO: Objetivando estudar os efeitos de lâminas de irrigação em populações de plantas da mamoneira BRS Energia, foi conduzido um experimento em esquema fatorial $4 \times 4$, sendo quatro lâminas de irrigação $(311,74 ; 460,40 ; 668,53$ e $850,54 \mathrm{~mm}$ ) e quatro populações de plantas $\left(27.777,33.333,41.666\right.$ e 55.555 plantas ha $\left.{ }^{-1}\right)$, com quatro repetições. O delineamento foi de blocos ao acaso com parcelas dispostas em faixas. O trabalho foi executado na Fazenda Experimental da Embrapa Algodão, localizada no município de Barbalha - CE. Por meio do método de irrigação por aspersão, estimaram-se as lâminas de acordo com a distância da linha central de aspersores em relação às faixas irrigadas. Na lâmina de 668,53 mm a BRS Energia obteve-se as principais respostas para altura de planta $(111,43 \mathrm{~cm})$; área foliar $\left(8.111,78 \mathrm{~cm}^{2}\right) \mathrm{e}$ produtividade $\left(2.678,38 \mathrm{~kg} \mathrm{ha}^{-1}\right)$. Na população de 33.333 plantas ha ${ }^{-1}$ verificou-se a maior taxa de crescimento absoluto $\left(1,99 \mathrm{~cm} \mathrm{dia}^{-1}\right)$, enquanto em 55.555 plantas ha $^{-1}$ deteve o maior índice de área foliar $\left(3,25 \mathrm{~m}^{2} \mathrm{~m}^{-2}\right)$. Todas as populações se comportaram semelhante quanto à produtividade.

PALAVRAS-CHAVE: Ricinus communis L., densidade de plantas, fisiologia das plantas.
ABSTRACT: Aiming to study the effects of depth of irrigation and plant density of castor bean BRS Energia, an experiment was carried out resulting from factorial combination $4 \times 4$, being four depths of irrigation water (311.74; 460.40; 668.53 and $850.54 \mathrm{~mm}$ ) and four plant densities (27.777, 33.333, 41.666 and 55,555 plants $\mathrm{ha}^{-1}$ ) with four repetitions. A randomized block design was used with plots in strips. The work was carried at Experimental Farm of Embrapa Algodão, located in the municipality of Barbalha - CE. Through sprinkler irrigation method, were estimated the depth of water applied were estimated in accordance with line source sprinkler irrigation system. In irrigation depth of $668.53 \mathrm{~mm}$ the castor bean BRS Energia corresponded to main response in plant height $(111.43 \mathrm{~cm})$; leaf area $\left(8,111.78 \mathrm{~cm}^{2}\right)$ and productivity $(2,678.40 \mathrm{~kg}$ $\left.h a^{-1}\right)$. In the population of 33,333 plants $\mathrm{ha}^{-1}$ highest absolute growth rate $\left(1.99 \mathrm{~cm} \mathrm{day}^{-1}\right)$ was observed, while leaf area index $\left(3.25 \mathrm{~m}^{2} \mathrm{~m}^{-2}\right)$ were verified in the population of 55,555 plants $\mathrm{ha}^{-1}$. All population behaved similarly in terms of productivity.

KEYWORDS: Ricinus communis L, density of plants, physiology of plants.

\section{Introdução}

O cultivo da mamoneira (Ricinus communis L.) no Brasil está em crescimento, principalmente no Nordeste (COSTA et al., 2009; BELTRÃO et al., 2010), o qual é responsável por aproximadamente $90 \%$ da produção nacional. A ideia de expandir a área de exploração de mamona está embasada principalmente na agricultura familiar para promover inclusão social e gerar emprego e renda neste setor, além de favorecer a redução de custos em relação ao petróleo (SANTOS et al., 2011).

No entanto, o grande desafio na região semiárida brasileira produtora de mamona é superar os baixos 
rendimentos médios alcançados nos cultivos, apesar dos incrementos ocorridos nos últimos anos. Neste sentido, a irrigação suplementar apresenta-se como prática eficiente no aumento da produtividade da cultura (SOUZA et al., 2007a; BISCARO et al., 2012), proporcionando aumento substancial nos números de cachos, frutos por planta e peso das sementes (BELTRÃO et al., 2003).

Outra maneira de aumentar a produtividade das culturas é o adensamento de plantas, mas o arranjo ideal destas na área de plantio depende de características intrínsecas de cada cultivar, como porte, hábito de crescimento e arquitetura da planta (BEZERRA et al., 2009).

A variação do adensamento populacional é uma prática simples e pode proporcionar aumento na produtividade em determinados genótipos, dependendo das condições de solo e clima (SEVERINO et al., 2006a; BIZINOTO et al., 2010).

Nesse contexto, o objetivo do presente trabalho foi avaliar o crescimento e desenvolvimento da mamoneira cv. BRS Energia quando submetidas a diferentes lâminas de água de irrigação e populações de plantas na Microrregião do Cariri no Estado do Ceará.

\section{Material e Métodos}

O trabalho foi desenvolvido com a mamoneira cv. BRS Energia no período de julho de 2006 a janeiro de 2007 na Fazenda Experimental da Embrapa Algodão, no município de Barbalha - CE, cujas coordenadas geográficas são: latitude $7^{\circ} 17^{\prime} 36,32^{\prime \prime} \mathrm{S}$, longitude $39^{\circ} 16$ ' 14,19" W e altitude de $387 \mathrm{~m}$. O clima predominante é Aw'/BSh, segundo classificação de Köppen, seco sub-úmido, com largo excesso hídrico no inverno. O solo da área experimental é um Neossolo Flúvico.

$\mathrm{O}$ delineamento foi em blocos ao acaso com parcelas dispostas dentro de faixas. Utilizaram-se dezesseis tratamentos resultantes da combinação fatorial de quatro lâminas de irrigação $\mathrm{L}_{1}-311,74 ; \mathrm{L}_{2}-460,40 ; \mathrm{L}_{3}-668,53$ e $\mathrm{L}_{4}-850,54 \mathrm{~mm}$, aplicadas a quatro populações $(\mathrm{P})$ de plantas espaçadas $0,6 \mathrm{~m}$ entre fileiras e $0,60,0,50,0,40$ e 0,30 m entre plantas, que contabilizaram na $\mathrm{P}_{1}-27.777, \mathrm{P}_{2}-33.333, \mathrm{P}_{3}-41.666$ e $\mathrm{P}_{4}-55.555$ plantas $\mathrm{ha}^{-1}$ e quatro repetições, totalizando 64 unidades experimentais.

Cada parcela experimental referente a um tratamento foi constituído de $3 \mathrm{~m}$ de largura por $8 \mathrm{~m}$ de comprimento totalizando $24 \mathrm{~m}^{2}$, o que permitiu a seguinte distribuição das plantas dentro das parcelas para os respectivos tratamentos em estudo: $\mathrm{P}_{1}-65, \mathrm{P}_{2}-80, \mathrm{P}_{3}-100$ e $\mathrm{P}_{4}-130$ plantas parcela ${ }^{-1}$. As parcelas tinham cinco fileiras, considerando como bordadura a primeira e a última linha de plantio.

As recomendações para adubação de NPK sugeridas com base nas análises químicas do solo (Tabela 1) foram $55-40-10 \mathrm{~kg} \mathrm{ha}^{-1}$ respectivamente.

$\mathrm{Na}$ metodologia de aplicação da adubação mineral, parcelou-se o nitrogênio em $1 / 3$ na fundação e $2 / 3$ no início da floração aos 40 dias após germinação (DAG); $\mathrm{P}_{2} \mathrm{O}_{5}$ foi aplicado exclusivamente na fundação, enquanto $\mathrm{o}_{2} \mathrm{~K}_{2} \mathrm{O}$ foi $2 / 3$ na fundação e $1 / 3$ no início da floração de acordo com a recomendação da Embrapa. Utilizou-se sulfato de amônio,
Tabela 1. Características químicas e físico-hídricas do solo da área experimental na profundidade de 0-20 cm - Barbalha, 2007.

\begin{tabular}{|c|c|}
\hline Características químicas & Unidades \\
\hline Cálcio $\left(\mathrm{cmol}_{\mathrm{c}} \mathrm{dm}^{-3}\right)$ & 8,63 \\
\hline Magnésio $\left(\mathrm{cmol}_{\mathrm{c}} \mathrm{dm}^{-3}\right)$ & 4,91 \\
\hline Sódio $\left(\mathrm{cmol}_{\mathrm{c}} \mathrm{dm}^{-3}\right)$ & 0,25 \\
\hline Potássio $\left(\mathrm{cmol}_{\mathrm{c}} \mathrm{dm}^{-3}\right)$ & 0,75 \\
\hline Hidrogênio $\left(\mathrm{cmol}_{\mathrm{c}} \mathrm{dm}^{-3}\right)$ & 0,00 \\
\hline Alumínio $\left(\mathrm{cmol}_{\mathrm{c}} \mathrm{dm}^{-3}\right)$ & 0,00 \\
\hline Soma de bases $(\mathrm{S})\left(\mathrm{cmol}_{\mathrm{c}} \mathrm{dm}^{-3}\right)$ & 14,54 \\
\hline CTC $\left(\mathrm{cmol}_{\mathrm{c}} \mathrm{dm}^{-3}\right)$ & 14,54 \\
\hline Saturação de bases (V), \% & 100 \\
\hline Matéria orgânica, $\mathrm{g} \mathrm{kg}^{-1}$ & 15,90 \\
\hline Fósforo, $\mathrm{mg} \mathrm{dm}^{-3}$ & 8,40 \\
\hline pH em água $(1: 2,5)$ & 7,30 \\
\hline $\mathrm{CE}$ do extrato de saturação, $\mathrm{dS} \mathrm{m}^{-1}$ & $0,13 *$ \\
\hline \multirow{2}{*}{ Classificação em relação à salinidade } & Não salino \\
\hline & Não sódico \\
\hline \multicolumn{2}{|l|}{ Características físico-hídricas* } \\
\hline Areia $(\%)$ & 64,06 \\
\hline Silte $(\%)$ & 4,02 \\
\hline Argila (\%) & 31,92 \\
\hline Classificação textural & Franco-Argilo-Arenoso \\
\hline Densidade aparente do solo, $\mathrm{kg} \mathrm{dm}^{-3}$ & 1,45 \\
\hline Densidade real, $\mathrm{kg} \mathrm{dm}^{-3}$ & 2,70 \\
\hline Porosidade Total \% & 46,29 \\
\hline Umidade -\% (0,33 atm) & 20,83 \\
\hline Umidade - \% (15 atm) & 8,83 \\
\hline Água disponível (\%) & 12,00 \\
\hline
\end{tabular}

fosfato monoamônico (MAP) e cloreto de potássio como fonte de nutrientes. Não foram feitas aplicações de herbicidas, inseticidas e fungicidas em nenhuma época, pois não foi necessário.

Nos primeiros vinte dias aplicou-se uma lâmina de irrigação uniforme em todos os tratamentos para garantir a germinação das plantas. Em seguida, utilizou-se o sistema 'line source sprinkler irrigation' proposto por Hanks et al. (1976). O controle da lâmina de irrigação foi uma média obtida através de testes no campo, aferindo-se as precipitações dos aspersores nas faixas em estudo, através de coletores, em relação à linha central.

As avaliações de crescimento e desenvolvimento foram realizadas a partir dos 20 dias após germinação (DAG) e em intervalos de 20 dias, sendo avaliadas as variáveis: altura da planta, área foliar, taxa de crescimento absoluto e relativo e 
índice de área foliar; acompanhando sempre as mesmas plantas, devidamente identificadas do início até o final do experimento, além da variável de produção, peso dos frutos totais, no final do experimento.

$\mathrm{Na}$ análise estatística para a variável altura de planta, utilizou-se o modelo sigmóide, conhecido também por regressão logística, conforme Equação 1.

$\mathrm{Y}_{\mathrm{i}}=\alpha /\left(1+e-{ }^{(\beta+\theta t \mathrm{ti})}\right)+\varepsilon_{\mathrm{i}}$

em que, $\alpha, \beta$ e $\theta$ são parâmetros a serem estimados, sendo $\alpha$ $>0$ e $\theta>0$; Y refere-se a variável em estudo e T o tempo (20, 40, 60, 80 e 100 DAG) da leitura (HOFFMANN; VIEIRA, 1998). As medições e determinações da área foliar foram realizadas conforme recomendação de Severino et al. (2004). $\mathrm{Na}$ análise da área foliar, utilizou-se o modelo de regressão polinomial cúbica, uma vez que, normalmente não se utiliza o modelo logístico para a área foliar devido à senescência foliar (CALBO; SILVA; TORRES, 1989).

Nas taxas de crescimento absoluto e relativo para altura da planta e índice de área foliar não foram realizadas análises de variância, devido a não recomendação estatística, pois são variáveis secundárias e contínuas, ou seja, derivadas da AP e AF (HOFFMANN; VIEIRA, 1998). Estas variáveis procuraram ajustar-se aos modelos de Regressão Exponencial permitindo que curvas descrevessem com maior exatidão possível a lei de respostas esperadas (hipótese), além de possuírem maiores coeficientes de determinação $\left(\mathrm{R}^{2}\right)$, onde todos os parâmetros do modelo fossem significativos até $5 \%$. Aplicou-se o software Tablecurve 2D para a construção dos gráficos.

Exceto as variáveis supracitadas, as demais foram realizadas mediante análise de variância, enquanto na variável de produção para os fatores lâminas de irrigação e população de plantas, por serem de natureza quantitativa, quando identificado efeito significativo, procedeu-se a análise de regressão polinomial.

\section{Resultados e Discussão}

\section{Altura de planta}

Nas análises de variância para a variável altura de planta (AP) identificaram-se efeitos significativos para o fator lâmina de irrigação (L) em nível de $(\mathrm{p}<0,01)$ a partir dos 40 DAG, porém não foram observados efeitos significativos para o fator população de plantas $(\mathrm{P})$ e também para a interação dos fatores $(\mathrm{L} \times \mathrm{P})$ em nenhuma das épocas estudadas (Tabela 2 ).

A altura de planta em função da lâmina de irrigação aos 20 DAG foi de aproximadamente $20 \mathrm{~cm}$ em média, não havendo diferenças significativas. A partir dos 40 DAG estimaram-se as seguintes AP, 45,97; 56,90; 61,20 e 48,89 $\mathrm{cm}$ para os tratamentos $\left(\mathrm{L}_{1}\right) ;\left(\mathrm{L}_{2}\right) ;\left(\mathrm{L}_{3}\right)$ e $\left(\mathrm{L}_{4}\right)$, respectivamente. Com estes resultados verificou-se que $\mathrm{L}_{1}$ deteve menor $\mathrm{AP}$, sendo inferior em $24,88 \%$ quando comparado a $\mathrm{L}_{3}$, já a $\mathrm{L}_{2} \operatorname{com} 7,02 \%$ e a $\mathrm{L}_{4}$ com 20,11\% também tiveram AP inferiores em relação $\mathrm{L}_{3}$ (Figura 1a). Aos $60 \mathrm{DAG} \mathrm{a} \mathrm{L}_{3}$ manteve seu comportamento com maior AP e os valores percentuais encontrados não variaram muito em relação aos obtidos na época anterior, com 26,79; 7,04 e 16,54\% de diminuição em $\mathrm{L}_{1}, \mathrm{~L}_{2}$ e $\mathrm{L}_{4}$ comparado a $\mathrm{L}_{3}$, respectivamente.

Aos 80 e 100 DAG as plantas de mamoneira estabilizaram seu crescimento e atingiram suas maiores alturas com 109,25 e $111,43 \mathrm{~cm} \mathrm{na} \mathrm{L}_{3}$, denotando para esta cultivar seu porte baixo, $\mathrm{o}$ que pode favorecer a um adensamento de plantas. Estes valores se aproximaram dos determinados por Silva et al. (2009) que em condições ambientais semelhantes obteve aos 100 DAG altura de planta com 105,74 cm.

Em relação ao fator população de plantas, a maior AP foi obtida na $\mathrm{P}_{1}$ (27.777 plantas ha-1) com 104,51 cm, não distante dos valores das outras populações (Figura 1b), implicando numa alta capacidade de adaptação da mamoneira nas mais variadas populações de plantas.

Tabela 2. Resumos das análises de variância da altura de planta (AP) da mamona irrigada com lâminas em diferentes populações aos $20,40,60,80$ e 100 dias após geminação (DAG) - Barbalha-CE, 2007.

\begin{tabular}{|c|c|c|c|c|c|c|}
\hline \multirow{2}{*}{ Causa de Variância } & \multicolumn{6}{|c|}{ Quadrados Médios } \\
\hline & GL & 20 DAG & 40 DAG & 60 DAG & 80 DAG & 100 DAG \\
\hline Blocos & 3 & 9,3781 & 123,7768 & 1369,4417 & 1732,8343 & 1259,1708 \\
\hline Lâmina (L) & 3 & $8,6461^{\text {ns }}$ & $893,0960 * *$ & $2062,2899 * *$ & $2353,8940 * *$ & $1586,4365^{* *}$ \\
\hline Resíduo 1 & 9 & 3,4585 & 123,9912 & 130,6344 & 84,8413 & 225,5934 \\
\hline População (P) & 3 & $1,6920^{\mathrm{ns}}$ & $93,7082^{\mathrm{ns}}$ & $242,7132^{\text {ns }}$ & $153,3795^{\mathrm{ns}}$ & $161,7888^{\mathrm{ns}}$ \\
\hline Resíduo 2 & 9 & 2,9414 & 121,9786 & 134,1840 & 184,7037 & 215,2250 \\
\hline Interação $\mathrm{L} \times \mathrm{P}$ & 9 & $3,4633^{\text {ns }}$ & $124,3278^{\mathrm{ns}}$ & $118,5619^{\text {ns }}$ & $174,4154^{\mathrm{ns}}$ & $109,0140^{\text {ns }}$ \\
\hline Resíduo 3 & 27 & 3,6148 & 67,7528 & 154,6959 & 197,6208 & 279,1939 \\
\hline Total & 63 & & & & & \\
\hline CV (1) \% & & 9,17 & 21,63 & 13,15 & 9,32 & 15,03 \\
\hline $\mathrm{CV}(2) \%$ & & 8,46 & 21,46 & 13,33 & 13,75 & 14,68 \\
\hline CV (3) $\%$ & & 9,38 & 15,99 & 14,31 & 14,22 & 16,69 \\
\hline
\end{tabular}

Significativo a $0,01(* *)$ de probabilidade; ${ }^{\text {ns }}$ não significativo. 


\section{Área foliar}

$\mathrm{Na}$ variável área foliar ( $\mathrm{AF})$ verificaram-se efeitos significativos em nível de $(p<0,05)$ no fator lâmina de irrigação (L) aos 20 DAG e significativo ( $<<0,01$ ) aos 40 e 60 DAG, não havendo efeitos nas demais épocas, conforme os resumos das análises de variância (Tabela 3). Para o fator população de plantas (P) houve variação apenas aos $60 \mathrm{DAG}$, significativo ao nível de $\mathrm{p}<0,01$. Enquanto na interação dos fatores $(\mathrm{L} \times \mathrm{P})$ não se identificaram efeitos significativos em nenhuma das épocas em estudo.

Diferentemente do que aconteceu na variável AP houve efeito significativo no fator lâmina de irrigação aos $20 \mathrm{DAG}$ para a AF. Os valores determinados foram 973,17; 933,69;
830,18 e $838,73 \mathrm{~cm}^{2}$ nas $\mathrm{L}_{1}, \mathrm{~L}_{2}, \mathrm{~L}_{3}$ e $\mathrm{L}_{4}$, respectivamente (Figura 2a).

Aos 40 DAG as plantas dispostas na $\mathrm{L}_{3}$ detiveram maior AF em relação às demais lâminas, onde foram observados nas $\mathrm{L}_{1}$, $\mathrm{L}_{2}$ e $\mathrm{L}_{4}$ reduções de 44,$24 ; 33,89$ e $68,78 \%$, respectivamente. Verificou-se que $\mathrm{a} \mathrm{L}_{1}$ comparada à avaliação anterior foi a mais afetada em curto espaço de tempo (20 dias), onde foi estimado $2.896,57 \mathrm{~cm}^{2}$, enquanto a $\mathrm{L}_{3}$ foi de $5.194,98 \mathrm{~cm}^{2}$.

Como nesta época foram aplicadas lâminas de irrigação em quantidades diferentes, devido os tratamentos, logo, o efeito encontrado para a $L_{1}$ foi causado pela restrição hídrica, limitando o tamanho das folhas. Pelo mesmo motivo aos 60 DAG a $\mathrm{L}_{1}$ continuou com AF comprometida.

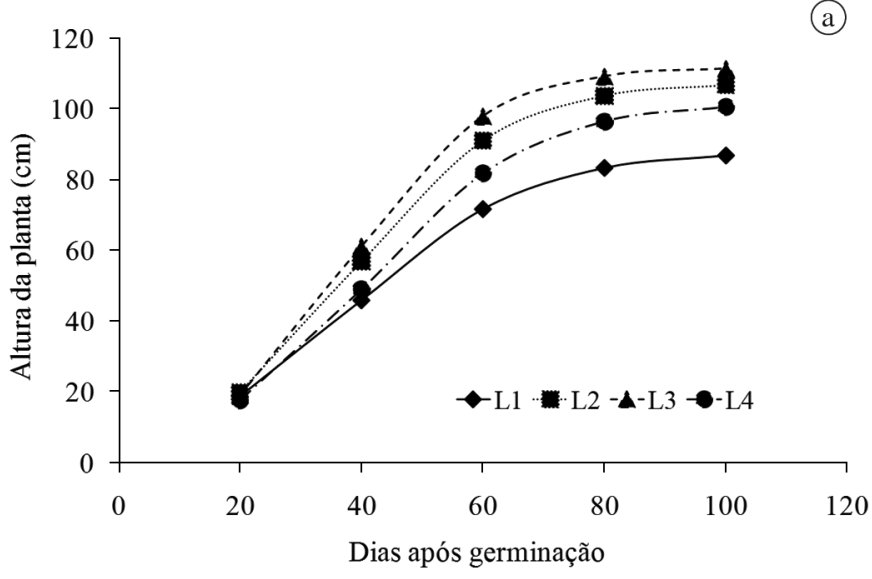

$(\mathrm{L} 1=311,74)=88,1497 /(1+\mathrm{e}(2,6937-0,0695 . \mathrm{t})) \quad \mathrm{R}^{2}=0,99$

$(\mathrm{L} 2=460,40)=107,600 /(1+\mathrm{e}(3,0724-0,0797 . \mathrm{t})) \quad \mathrm{R}^{2}=0,99$

$(\mathrm{L} 3=668,53)=111,900 /(1+\mathrm{e}(3,3436-0,0883 . \mathrm{t})) \quad \mathrm{R}^{2}=0,99$

$(\mathrm{L} 4=840,54)=101,000 /(1+\mathrm{e}(3,0486-0,0742 . \mathrm{t})) \quad \mathrm{R}^{2}=0,99$ (a)

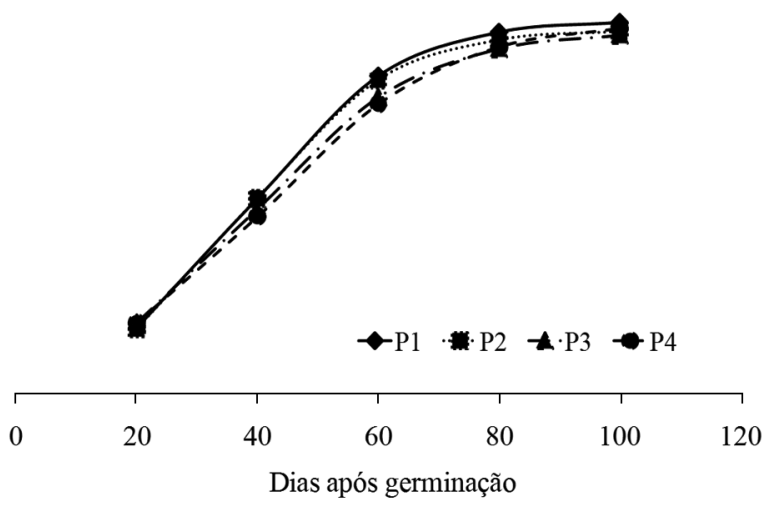

$(\mathrm{P} 1=27.777)=105,200 /(1+\mathrm{e}(3,2070-0,0823 . \mathrm{t})) \quad \mathrm{R}^{2}=0,99$

$(\mathrm{P} 2=33.333)=102,400 /(1+\mathrm{e}(3,2237-0,0842 . \mathrm{t})) \quad \mathrm{R}^{2}=0,99$

$(\mathrm{P} 3=41.666)=101,900 /(1+\mathrm{e}(2,8884-0,0730 . \mathrm{t})) \quad \mathrm{R}^{2}=0,99$

$(\mathrm{P} 4=55.555)=104,700 /(1+\mathrm{e}(2,8018-0,0675 . \mathrm{t})) \quad \mathrm{R}^{2}=0,99$

Figura 1. Altura da planta (AP) da mamona sob diferentes lâminas de irrigação (a) e população de plantas (b) em função dos dias após geminação (DAG) - Barbalha-CE, 2007.

Tabela 3. Resumos das análises de variância da área foliar (AF) da mamona irrigada com lâminas em diferentes populações aos $20,40,60,80$ e 100 dias após geminação (DAG) - Barbalha-CE, 2007.

\begin{tabular}{|c|c|c|c|c|c|c|}
\hline \multirow{2}{*}{$\begin{array}{c}\text { Causa de } \\
\text { Variância }\end{array}$} & \multirow{2}{*}{ GL } & \multicolumn{5}{|c|}{ Quadrados Médios } \\
\hline & & 20 & 40 & 60 & 80 & 100 \\
\hline Blocos & 3 & 18556,61 & 1789126,34 & 45488011,87 & 25832500,44 & 10456014,33 \\
\hline Lâmina (L) & 3 & $187582,05^{*}$ & $5972548,42 * *$ & $71105672,01 * *$ & $9022380,59^{\text {ns }}$ & $14908788,98^{\text {ns }}$ \\
\hline Resíduo 1 & 9 & 35776,00 & 710191,74 & 5674262,04 & 9546828,86 & 9556165,63 \\
\hline População (P) & 3 & $149329,34^{\mathrm{ns}}$ & $249170,59^{\mathrm{ns}}$ & $42915153,11^{* *}$ & $22613875,99^{\mathrm{ns}}$ & $6750764,31^{\mathrm{ns}}$ \\
\hline Resíduo 2 & 9 & 85001,60 & 603074,67 & 6517434,00 & 17451854,82 & 5539985,76 \\
\hline Interação L x P & 9 & $8809,52^{\text {ns }}$ & $521675,80^{\text {ns }}$ & $11994801,18^{\mathrm{ns}}$ & $9276973,48^{\mathrm{ns}}$ & $3236874,43^{\text {ns }}$ \\
\hline Resíduo 3 & 27 & 53721,96 & 737833,46 & 5991217,80 & 11392645,52 & 8749711,71 \\
\hline Total & 63 & & & & & \\
\hline CV (1)\% & & 19,56 & 24,36 & 35,42 & 47,04 & 78,25 \\
\hline CV (2)\% & & 30,14 & 22,44 & 37,96 & 63,60 & 59,58 \\
\hline $\mathrm{CV}(3) \%$ & & 23,96 & 24,83 & 36,39 & 51,38 & 74,87 \\
\hline
\end{tabular}

Significativo a $0,05(*)$ e a $0,01(* *)$ de probabilidade; ns não significativo. 


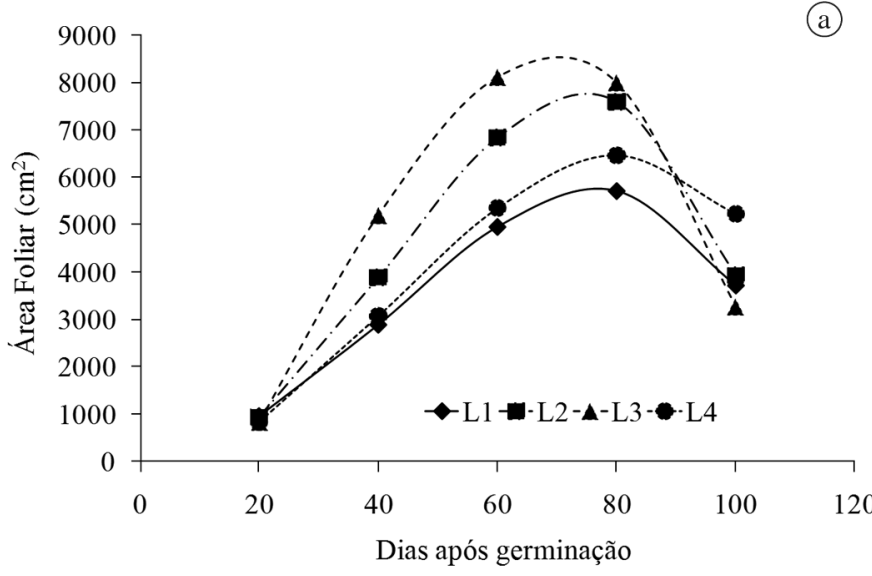

$\begin{array}{ll}(\mathrm{L} 1=311,74)=625,77-46,03 \mathrm{x}+3,77 \mathrm{x}^{2}-0,030 \mathrm{x}^{3} & \mathrm{R}^{2}=0,99 \\ (\mathrm{~L} 2=460,40)=203,29-55,68 \mathrm{x}+5,53 \mathrm{x}^{2}-0,046 \mathrm{x}^{3} & \mathrm{R}^{2}=0,99 \\ (\mathrm{~L} 3=668,53)=-3398,62+181,64 \mathrm{x}+2,15 \mathrm{x}^{2}-0,033 \mathrm{x}^{3} & \mathrm{R}^{2}=0,91 \\ (\mathrm{~L} 4=850,54)=-160,47-1,04 \mathrm{x}+3,05 \mathrm{x}^{2}-0,025 \mathrm{x}^{3} & \mathrm{R}^{2}=0,99\end{array}$ (a)

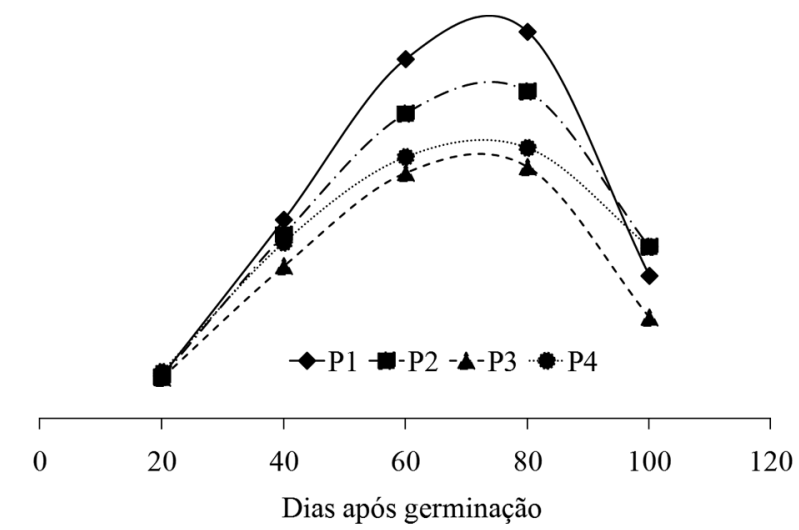

$(\mathrm{P} 1=27.777)=591,82-108,30 \mathrm{x}+7,32 \mathrm{x}^{2}-0,060 \mathrm{x}^{3} \quad \mathrm{R}^{2}=0,94$ $(\mathrm{P} 2=33.333)=-913,60+31,88 \mathrm{x}+3,53 \mathrm{x}^{2}-0,034 \mathrm{x}^{3} \quad \mathrm{R}^{2}=0,98$ $(\mathrm{P} 3=41.666)=-435,39+13,33 \mathrm{x}+3,12 \mathrm{x}^{2}-0,030 \mathrm{x}^{3} \quad \mathrm{R}^{2}=0,99$ $(\mathrm{P} 4=55.555)=-1983,09+142,53 \mathrm{x}+0,53 \mathrm{x}^{2}-0,014 \mathrm{x}^{3} \quad \mathrm{R}^{2}=0,99$

Figura 2. Área foliar (AF) da mamona sob diferentes lâminas de irrigação (a) e população de plantas (b) em função dos dias após geminação (DAG) Barbalha-CE, 2007.

Resultados semelhantes foram encontrados por Lacerda, Guerra e Barros Junior (2009) que verificaram superioridade nas $\mathrm{AF}$, cujo conteúdo de água no solo foi mantido na capacidade de campo (100\% água disponível-AD) em relação aos tratamentos 70, 80 e $90 \% \mathrm{AD}$, principalmente aos $70 \%$ $\mathrm{AD}$ em 37,6\%. Rodrigues et al. (2009), por sua vez, observou que plantas irrigadas com 0,6 ETc em relação a 1,20 ETc, tiveram AF inferiores em 178,87, 47,15 e 115,98\%, aos 48, 90 e 132 dias após semeadura.

Para o fator população de plantas, a $\mathrm{P}_{1}$ destacou-se com maior área foliar aos $60 \mathrm{DAG}$, enquanto a $\mathrm{P}_{2}, \mathrm{P}_{3}$ e $\mathrm{P}_{4}$ foram inferiores em 17,$64 ; 46,31$ e $37,28 \%$, respectivamente (Figura 2b). Estes resultados implicaram numa melhor adaptação da $\mathrm{P}_{1}$, provavelmente devido a não competição por água, luz e nutrientes a que as plantas de mamoneira das outras populações foram submetidas. Além de interferências das folhas superiores sobre as inferiores de plantas muito adensadas $\left(\mathrm{P}_{3}\right.$ e $\left.\mathrm{P}_{4}\right)$, provocando a diminuição da área foliar a partir de determinada fase fenológica da cultura.

\section{Taxas de crescimento absoluto e relativo da altura da planta}

Analisando a $\mathrm{TCA}_{\mathrm{AP}}$ em função da lâmina de irrigação verificou-se que no intervalo 20-40 DAG foram estimados os seguintes valores 1,$27 ; 1,71 ; 1,99$ e $1,32 \mathrm{~cm} \mathrm{dia}^{-1}$, inferindo a $\mathrm{L}_{3}(668,53 \mathrm{~mm})$ maior desempenho.

Notadamente no intervalo 40-60 DAG as plantas atingiram suas maiores atividades metabólicas contribuindo para as maiores $\mathrm{TCA}_{\mathrm{AP}}$ em quase todas as lâminas com 1,35; 1,82; 1,94 e $1,85 \mathrm{~cm} \mathrm{dia}^{-1}$. Neste intervalo, verificou-se um expressivo aumento na $\mathrm{L}_{4}$, como também foi observado diretamente na variável AP para as respectivas épocas.

Rodrigues et al. (2008), determinaram maior taxa de crescimento diário entre 48 e 90 DAS, com média de
$1,36 \mathrm{~cm} \mathrm{dia}{ }^{-1}$ utilizando águas residuárias, na cultivar BRS Paraguaçu. Para tanto, os valores da TCA $\mathrm{AP}_{\mathrm{P}}$ no presente estudo na menor lâmina foram próximos deste último e esta diferença pode estar relacionada com o fato de ter sido estudado outra cultivar de mamoneira, onde seu ciclo é bem mais curto, permitindo determinar estes resultados superiores.

Para os intervalos 60-80 e 80-100 DAG houve uma diminuição acentuada e uma estabilização para a TCA ${ }_{\mathrm{AP}}$, sendo um comportamento semelhante entre as plantas de todos os tratamentos (Figura 3a). De maneira similar também foi verificado pelos autores acima citados que no final de seu experimento não se constatou diferença significativa entre aquelas cultivares estudadas.

Considerando a taxa de crescimento absoluto em função da população de plantas, observou-se a mesma tendência que encontrado em relação à lâmina de irrigação, ou seja, nos intervalos 20-40 e 40-60 DAG, as plantas tiveram as maiores TCAap, diminuindo nos intervalos seguintes. Mediante o exposto na Figura 3b, no intervalo 40-60 DAG foi encontrado os maiores valores desta variável, cujas populações mais numerosas apresentaram menor desenvolvimento.

Nos intervalos seguintes as leituras foram equiparadas e o fato de estar próximo do final do ciclo da cultura, foi fator determinante para as reduções nas $\mathrm{TCA}_{\mathrm{AP}}$. Para Larcher (2000), paralisações no crescimento vegetativo em função da aceleração do crescimento produtivo, como nas últimas épocas verificadas, ocorreram pela canalização da energia de nutrientes e assimilados destinados à floração e frutificação.

Considerando a taxa de crescimento relativo para altura de planta $\left(\mathrm{TCR}_{\mathrm{AP}}\right)$ nas diferentes lâminas de irrigação e populações de plantas da mamoneira em função do intervalo de tempo, também se observou valores aproximados e as características do crescimento das plantas em termos de ganho de altura se deram mais nitidamente nos intervalos 20-40 e 40-60 DAG, 
enquanto nos intervalos 60-80 e 80-100 DAG não foram observados efeitos significativos.

As maiores TCR $\mathrm{AP}_{\mathrm{P}}$ ocorreram no $1^{\mathrm{o}}$ intervalo, sendo identificado na $\mathrm{L}_{3}$ e $\mathrm{P}_{1}=0,05 \mathrm{~cm} \mathrm{~cm}^{-1} \mathrm{dia}^{-1}$ (Figuras $4 \mathrm{a}, \mathrm{b}$ ) que seguiu de forma semelhante o comportamento no $\mathrm{TCA}_{\mathrm{AP}}$ No intervalo 40-60 DAG tanto a $\mathrm{L}_{3}$ como a $\mathrm{P}_{1}$ continuaram com maiores taxas, porém as demais lâminas de irrigação e populações de plantas mantiveram-se próximas. Nos intervalos seguintes verificou-se uma redução na taxa de crescimento relativo para os dois fatores.

Estes resultados se aproximaram dos obtidos por Rodrigues et al. (2008), que verificaram para a avaliação entre 48 e 90 DAS que em todos os níveis de reposição de águas residuárias a $\mathrm{TCR}_{\mathrm{AP}}$ se aproximou de $0,02 \mathrm{~cm} \mathrm{~cm}^{-1} \mathrm{dia}^{-1}$, o qual, foi a média para a BRS Nordestina e BRS Paraguaçu. A partir desta época ocorreram reduções no $\mathrm{TCR}_{\mathrm{AP}}$, seguindo o comportamento das demais variáveis.

\section{Índice de área foliar}

No fator lâmina de irrigação, observou-se que os resultados encontrados aos 20 DAG foram próximos entre si e seguiu o mesmo comportamento da AF. Aos 40, 60 e 80 DAG, determinaram-se respectivamente os seguintes valores para $\mathrm{L}_{1} ; 1,08 ; 1,84$ e $2,18 \mathrm{~m}^{2} \mathrm{~m}^{-2}$, os quais foram os menores IAF nas plantas de mamoneira cv. BRS Energia.

$\mathrm{Na}_{3}$ foram verificados os maiores valores 1,$86 ; 3,25 \mathrm{e}$ $3,13 \mathrm{~m}^{2} \mathrm{~m}^{-2}$ nas mesmas datas da $\mathrm{L}_{1}$ (Figura 5a). $\mathrm{Na} \mathrm{L}_{2}$ as leituras foram sempre as mais aproximadas da $\mathrm{L}_{3}$, enquanto

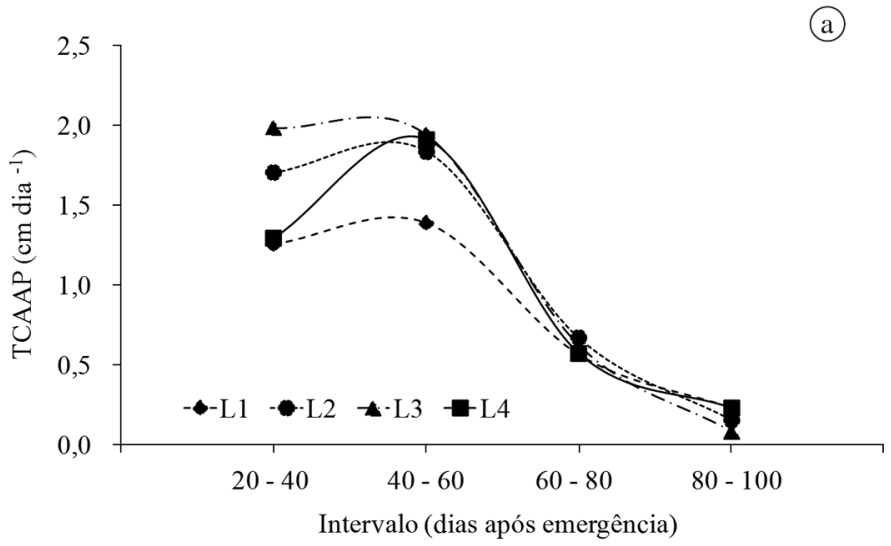

(b)

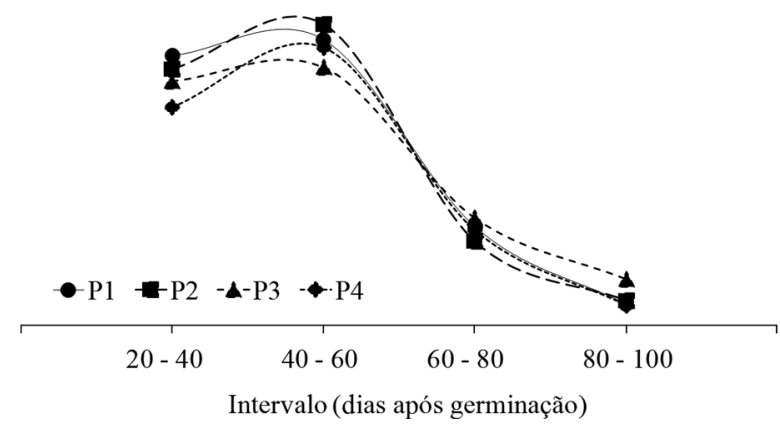

$(\mathrm{L} 1=311,74)=\operatorname{EXP}\left(-0,5717+1,1889 \mathrm{x} * *-0,3765 \mathrm{x}^{2 * *}\right) \quad \mathrm{R}^{2}=0,98$

$(\mathrm{P} 1=27.777)=\operatorname{EXP}\left(-0,5601+1,6164 \mathrm{x}^{* *}-0,5227 \mathrm{x}^{2 * *}\right) \quad \mathrm{R}^{2}=0,99$ $(\mathrm{L} 2=460,40)=\operatorname{EXP}\left(-0,5265+1,5626 \mathrm{x}^{* *}-0,4998 \mathrm{x}^{2 * *}\right) \quad \mathrm{R}^{2}=0,99$ $(\mathrm{P} 2=33.333)=\operatorname{EXP}\left(-0,8490+1,9377 \mathrm{x}^{* *}-0,5999 \mathrm{x}^{2 * *}\right) \quad \mathrm{R}^{2}=0,99$ $(\mathrm{P} 3=41.666)=\operatorname{EXP}\left(-0,2443+1,0295 \mathrm{x}^{* *}-0,3396 \mathrm{x}^{2 * *}\right) \quad \mathrm{R}^{2}=0,98$ $(\mathrm{L} 3=668,53)=\operatorname{EXP}\left(-0,3948+1,6336 \mathrm{x}^{* *}-0,5519 \mathrm{x}^{2 * *}\right) \quad \mathrm{R}^{2}=0,99$ $(\mathrm{L} 4=850,54)=\operatorname{EXP}\left(-1,2151+2,0736 \mathrm{x} * *-0,5787 \mathrm{x}^{2 * *}\right) \quad \mathrm{R}^{2}=0,95$

$(\mathrm{P} 4=55.555)=\operatorname{EXP}\left(-1,1302+2,0671 \mathrm{x}^{* *}-0,6123 \mathrm{x}^{2 * *}\right) \quad \mathrm{R}^{2}=0,99$

Figura 3. Taxa de crescimento absoluto da altura da planta $\left(\mathrm{TCA}_{\mathrm{AP}}\right)$ da mamona sob diferentes lâminas de irrigação (a) e população de plantas (b) em função dos dias após geminação (DAG) - Barbalha-CE, 2007.

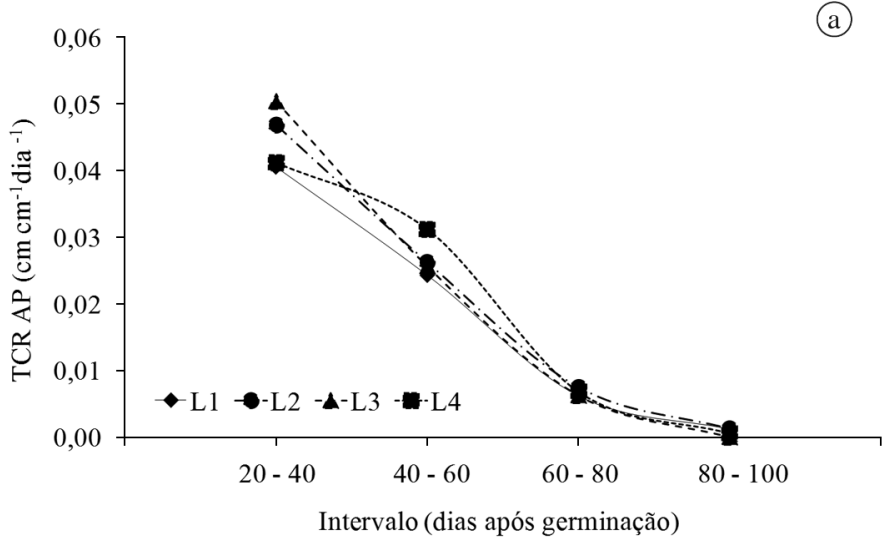

$(\mathrm{L} 1=311,74)=\operatorname{EXP}\left(-3,4790+0,6714 \mathrm{x}^{* *}-0,3954 \mathrm{x}^{2 * *}\right) \quad \mathrm{R}^{2}=0,99$ $(\mathrm{L} 2=460,40)=\operatorname{EXP}\left(-3,1422+0,4134 \mathrm{x}^{* *}-0,3313 \mathrm{x}^{2 * *}\right) \quad \mathrm{R}^{2}=0,99$ $(\mathrm{L} 3=668,53)=\operatorname{EXP}\left(-2,8182+0,2356 \mathrm{x}^{* *}-0,3292 \mathrm{x}^{2 * *}\right) \quad \mathrm{R}^{2}=0,99$ $(\mathrm{L} 4=850,54)=\operatorname{EXP}\left(-4,1375+1,5632 \mathrm{x}^{* *}-0,6138 \mathrm{x}^{2 * *}\right) \quad \mathrm{R}^{2}=0,99$ (a)

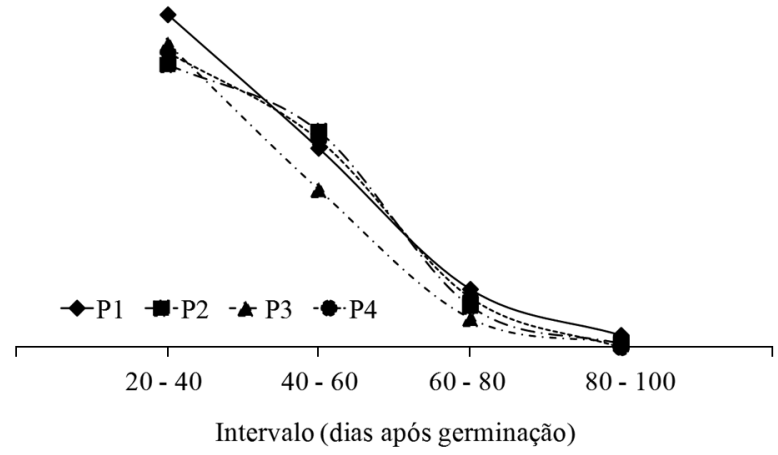

$(\mathrm{P} 1=27.777)=\operatorname{EXP}\left(-2,0733+0,9194 \mathrm{x} * *-0,0014 \mathrm{x}^{2 * *}\right) \quad \mathrm{R}^{2}=0,99$ $(\mathrm{P} 2=33.333)=\operatorname{EXP}\left(-4,2478+1,7691 \mathrm{x}^{* *}-0,6795 \mathrm{x}^{2 * *}\right) \quad \mathrm{R}^{2}=0,99$ $(\mathrm{P} 3=41.666)=\operatorname{EXP}\left(-3,4284+0,8390 \mathrm{x} * *-0,4978 \mathrm{x}^{2 * *}\right) \quad \mathrm{R}^{2}=0,98$ $(\mathrm{P} 4=55.555)=\operatorname{EXP}\left(-3,8409+1,2640 \mathrm{x}^{* *}-0,5382 \mathrm{x}^{2 * *}\right) \quad \mathrm{R}^{2}=0,99$

Figura 4. Taxa de crescimento relativo da altura da planta $\left(\mathrm{TCR}_{\mathrm{AP}}\right)$ da mamona sob diferentes lâminas de irrigação (a) e população de plantas (b) em função dos dias após geminação (DAG) - Barbalha-CE, 2007. 

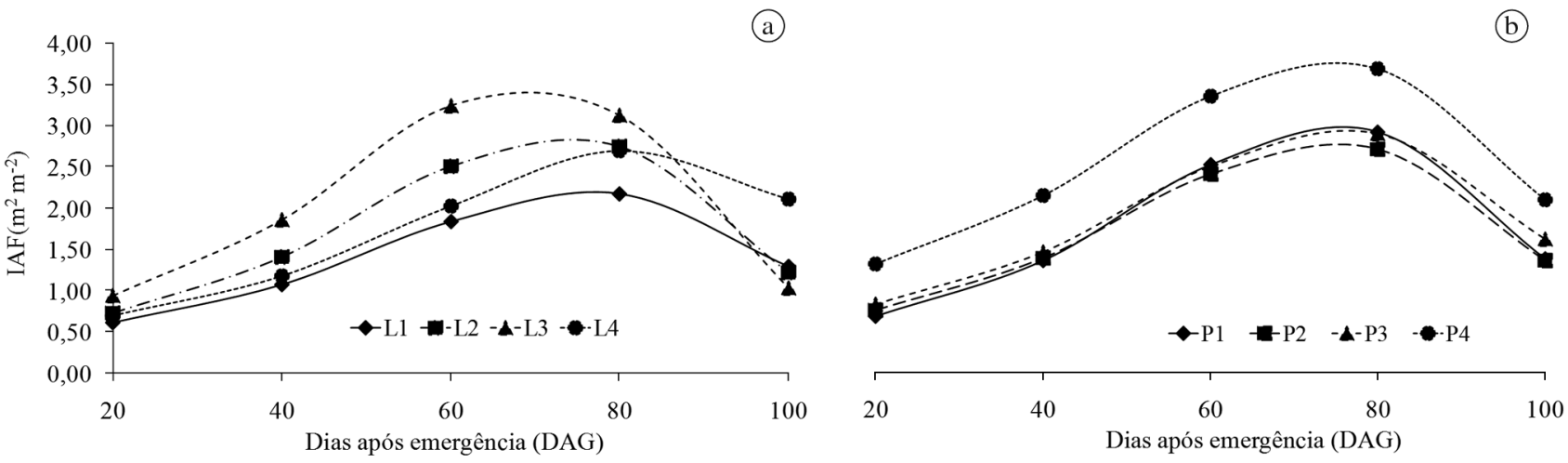

$(\mathrm{L} 1=311,74)=\operatorname{EXP}\left(-0,7328+0,00078 \mathrm{x}^{2}-6,8.10^{-6} \mathrm{x}^{3}\right) \quad \mathrm{R}^{2}=0,96$ $(\mathrm{L} 2=460,40)=\operatorname{EXP}\left(-0,6155+0,00095 \mathrm{x}^{2}-8,6.10^{-6} \mathrm{x}^{3}\right) \quad \mathrm{R}^{2}=0,99$ $(\mathrm{L} 3=668,53)=\operatorname{EXP}\left(-0,3906+0,00102 \mathrm{x}^{2}-9,8 \cdot 10^{-6} \mathrm{x}^{3}\right) \quad \mathrm{R}^{2}=0,99$ $(\mathrm{L} 4=850,54)=\operatorname{EXP}\left(-0,5974+0,00070 \mathrm{x}^{2}-5,7.10^{-6} \mathrm{x}^{3}\right) \quad \mathrm{R}^{2}=0,95$

$(\mathrm{P} 1=27.777)=\operatorname{EXP}\left(-0,8195+0,00097 \mathrm{x}^{2}-8,7.10^{-6} \mathrm{x}^{3}\right) \quad \mathrm{R}^{2}=0,99$ $(\mathrm{P} 2=33.333)=\operatorname{EXP}\left(-0,6905+0,00087 \mathrm{x}^{2}-7,8.10^{-6} \mathrm{x}^{3}\right) \quad \mathrm{R}^{2}=0,99$ $(\mathrm{P} 3=41.666)=\operatorname{EXP}\left(-0,5837+0,00081 \mathrm{x}^{2}-7,2 \cdot 10^{-6} \mathrm{x}^{3}\right) \quad \mathrm{R}^{2}=0,98$ $(\mathrm{P} 4=55.555)=\operatorname{EXP}\left(-0,0836+0,00071 \mathrm{x}^{2}-6,4 \cdot 10^{-6} \mathrm{x}^{3}\right) \quad \mathrm{R}^{2}=0,99$

Figura 5. Índice de área foliar (IAF) da mamona sob diferentes lâminas de irrigação (a) e população de plantas (b) em função dos dias após geminação (DAG) - Barbalha-CE, 2007.

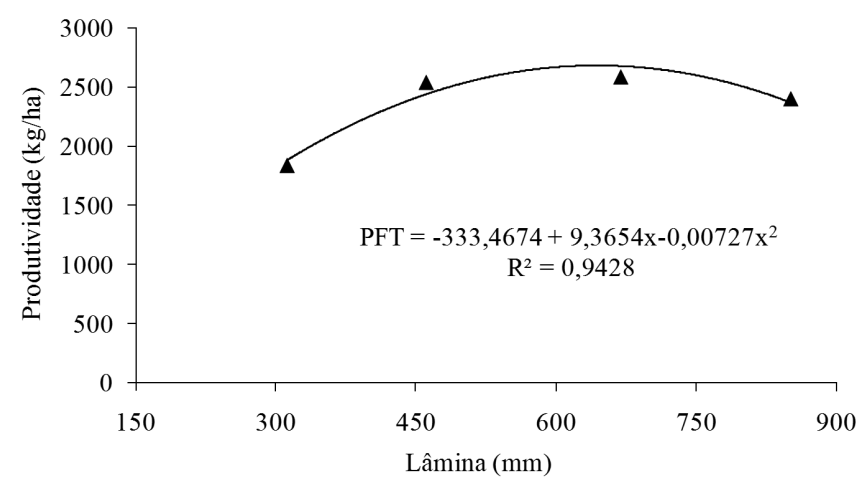

Figura 6. Peso dos frutos totais da mamona em função da lâmina de irrigação - Barbalha-CE, 2007.

Tabela 4. Resumo da análise de variância referente a variável peso dos frutos totais (PFT) da mamona irrigada com diferentes lâminas em diferentes populações de plantas - Barbalha-CE, 2007.

\begin{tabular}{lcc}
\hline \multirow{2}{*}{ Causa de Vriância } & \multicolumn{2}{c}{ Quadrados Médios } \\
\cline { 2 - 3 } & GL & PFT \\
\hline Blocos & 3 & 2564817,9173 \\
Lâmina (L) & 3 & $1896190,1803^{*}$ \\
Resíduo 1 & 9 & 319802,5981 \\
População (P) & 3 & $107038,5848^{\text {ns }}$ \\
Resíduo 2 & 9 & 370487,0389 \\
Interação L $\times$ P & 9 & $253627,4578^{\text {ns }}$ \\
Resíduo 3 & 27 & 437139,1945 \\
Total & 63 & 24,17 \\
CV (1)\% & & 26,01 \\
CV (2)\% & & 28,26 \\
CV (3) \% & &
\end{tabular}

* Significativo a 0,05 , ns não significativo. na $\mathrm{L}_{4}$ com o passar do tempo, os decréscimos foram menores. $\mathrm{A}_{3}$ foi em média superior em relação a $\mathrm{L}_{1}, \mathrm{~L}_{2}$ e $\mathrm{L}_{4}$ em 53,73; 55,40 e $24,44 \%$ para aquelas respectivas épocas.

Para o fator população de plantas tem-se em todas as datas que a $\mathrm{P}_{4}\left(55.555\right.$ plantas $\left.\mathrm{ha}^{-1}\right)$ deteve o maior IAF, o que é bastante compreensível pelo fato de contar a maior população de plantas de mamoneira. Aos 20 DAG, por exemplo, houve uma superioridade $>90 \%$ em relação à menor população.

Nas leituras de 60 e 80 DAG determinaram-se as menores diferenças percentuais das demais populações em relação a $\mathrm{P}_{4}$. Precisamente aos 80 DAG a mamoneira cultivar BRS Energia atingiu os maiores IAF com 2,58; 2,39; 2,56 e $3,25 \mathrm{~m}^{2} \mathrm{~m}^{-2}$ ratificando a $\mathrm{P}_{4}$ com valores mais expressivos. Aos 100 DAG, ficou evidente a queda das folhas e dos pecíolos, observando-se uma redução acentuada do IAF em todas as populações.

\section{Peso dos frutos totais}

Verificou-se efeito significativo apenas para lâmina de irrigação $(p<0,05)$ para o peso dos frutos totais $(\mathrm{PFT})$ que segue na Tabela 4. A variável foi submetida à regressão polinomial e o modelo matemático que melhor representou seu comportamento foi à função quadrática.

Os resultados estimados para a variável PFT foram os seguintes $1.879,59 ; 2.437,35 ; 2.678,38$ e $2.372,92 \mathrm{~kg} \mathrm{ha}^{-1} \mathrm{de}$ frutos para as lâminas 311,74; 460,40; 668,53 e 850,54 mm, respectivamente (Figura 6). A partir destes resultados, confirmou-se as respostas obtidas durante todo o estudo que a deficiência de água no início do crescimento (20 DAG) até a maturação dos frutos, levou a perdas consideráveis na produção da mamoneira para a cultivar BRS Energia, com redução nos percentuais na $L_{1}$ de $42,49 \%$ em relação a $L_{3}$.

Estes resultados corroboram com Diniz Neto et al. (2009) em Limoeiro do Norte-CE, ao verificar que a menor quantidade de água disponível às plantas no cultivo de sequeiro, afetou as estruturas vegetativas e reprodutivas da mamoneira. 
Já na $\mathrm{L}_{4}$ verificou-se recuperação dos percentuais, embora houvesse redução da produtividade de $12,87 \%$, levando a entender que a cultura é favorecida ao aumento da disponibilidade água, no início da fase de produção de frutos (BARROS JÚNIOR et al., 2008). Mas para Severino et al. (2006b), quando se tem um pequeno excesso de umidade no solo, há uma tendência excessiva do crescimento vegetativo das plantas de mamoneira, podendo comprometer a produção da cultura, o que pode ter ocorrido neste estudo. Já para Souza et al. (2007b), a suplementação hídrica é mais vantajosa no início do crescimento da cultura.

Quanto aos resultados da $\mathrm{L}_{2}$, verificou-se aproximação em relação a $L_{3}$, porém com uma redução de $9,88 \%$ na PFT, todavia é importante observar a economia de água, pois quando comparado às duas lâminas; $\mathrm{L}_{2}(460,40 \mathrm{~mm})$ e $\mathrm{L}_{3}(668,53 \mathrm{~mm})$ têm-se uma diferença de $208,13 \mathrm{~mm}$, que em se tratando da Região Nordeste, este primeiro valor se enquadra dentro da pluviometria de várias Mesorregiões.

No fator população de plantas não foi identificado efeito significativo para a variável PFT, ou seja, a produtividade da mamoneira BRS Energia foi semelhante quando submetidas às variações de populações de plantas. Os valores foram os seguintes; 2.282,74, 2.429,59, 2.259,29 e 2.387,43 $\mathrm{kg} \mathrm{ha}^{-1} \mathrm{de}$ frutos em 27.777; 33.333; 41.666 e 55.555 plantas ha $^{-1}$.

Diferentemente do encontrado por Diniz, Távora e Diniz Neto (2009) em seu estudo com densidades populacionais com a mamoneira, pois no espaçamento $2,0 \mathrm{~m} \times 1,0 \mathrm{~m}$ obteve rendimento de grãos de $1.470,99 \mathrm{~kg} \mathrm{ha}^{-1}$, superior ao espaçamento $2,0 \mathrm{~m} \times 2,0 \mathrm{~m}$ que obteve rendimento de $1.184,45 \mathrm{~kg} \mathrm{ha}^{-1}$, ou seja, aumentando o número de plantas atingiu maior produtividade. Carvalho (2010), estudando o espaçamento das cultivares Nordestina e Paraguaçu, também obteve maior produtividade com aumento da população de plantas.

\section{Conclusões}

1 - A lâmina de $668,53 \mathrm{~mm}$ obteve os melhores resultados em todas as variáveis estudadas.

2 - A variação na população de plantas, não influenciou significativamente a cultura da mamoneira, exceto na área foliar.

3 - Em todo o estudo o efeito interativo entre lâmina de irrigação e população de plantas não foi significativo induzindo que estes fatores se comportaram de maneira independente.

4 - O aumento na lâmina de irrigação no período de floração e frutificação da mamoneira favorece ao aumento da produção.

\section{Referências}

BARROS JÚNIOR, G. et al. Consumo de água e eficiência de uso para duas cultivares de mamona, submetidas a estresse hídrico. Revista Brasileira de Engenharia Agrícola e Ambiental, Campina Grande, v. 12, n. 4, p. 350-355, 2008. dx.doi.org./ 10.1590/ S1415-43662008000400003
BELTRÃO, N. E. M. et al. Fisiologia da mamoneira, cultivar BRS 149 Nordestina na fase inicial de crescimento, submetida a estresse hídrico. Revista Brasileira de Oleaginosas e Fibrosas, Campina Grande, v. 7, n. 1, p. 659-664, 2003.

BELTRÃO, N. E. M. et al. Consórcio mamona e amendoim: opção para a agricultura familiar. Revista Verde de Agroecologia e Desenvolvimento Sustentável, Mossoró, v. 5, n. 4, p. 222-227, 2010.

BEZERRA, A. A. C. et al. Características de dossel e de rendimento em feijão-caupi ereto em diferentes densidades populacionais. Pesquisa Agropecuária Brasileira, Brasília, v. 44, n. 10, p. 1239-1245, 2009. http://dx.doi.org/10.1590/S0100-204X2009001000005

BISCARO, A. G. et al. Produtividade de cultivares de mamona submetidas a diferentes lâminas de irrigação suplementar. Revista Brasileira de Engenharia Agrícola e Ambiental, Campina Grande, v. 16, n. 9, p. 925-930, 2012. dx.doi.org./ 10.1590/ S1415-43662012000900001

BIZINOTO, T. K. M. C. et al. Cultivo da mamoneira influenciada por diferentes populações de plantas. Bragantia, Campinas, v. 69 , n. 2 , p. 367-370, 2010. http://dx.doi.org/10.1590/S000687052010000200014

CAlBO, A. G.; SILVA, W. L. C.; TORRES, A. C. Comparação de modelos e estratégias para análise de crescimento. Revista Brasileira de Fisiologia Vegetal, Brasília, v. 1, n. 1, p. 1-7, 1989.

CARVALHO, E. V. Densidade de plantio de duas cultivares de mamona no Sul de Tocantins. Revista Ciência Agronômica, Fortaleza, v. 41, n. 3, p. 387-392, 2010. http://dx.doi.org/10.1590/ S1806-66902010000300010

COSTA, F. X. et al. Efeito do lixo orgânico e torta de mamona nas características de crescimento da mamoneira (Ricinus communis L.). Revista Engenharia Ambiental, Espírito Santo do Pinhal, v. 6, n. 1, p. 259-268, 2009.

DINIZ, B. L. M. T.; TÁVORA, F. J. A. F.; DINIZ NETO, M. A. Manipulação do crescimento da mamoneira através da poda em diferentes densidades populacionais. Revista Ciência Agronômica Fortaleza, v. 40, n. 4, p. 570-577, 2009.

DINIZ NETO, M. A. et al. Adubação NPK e épocas de plantio para a mamoneira - II Componentes das fases vegetativas e reprodutivas. Revista de Ciência Agronômica, Fortaleza, v. 40, n. 3, p. 417-426, 2009.

HANKS, R. J. et al. Line source sprinkler for continuous variable irrigation crop production studies. Soil Science Society of America Journal, Madison, v. 40, n. 3, p. 426-429, 1976.

HOFFMANN, R.; VIEIRA, S. Análise de regressão: uma introdução a econometria. 3. ed. São Paulo: Hucitec, 1998. 379 p.

LACERDA, R. D.; GUERRA, H. O. C.; BARROS JUNIOR, G. Influência do déficit hídrico e da matéria orgânica do solo no crescimento da mamoneira BRS 188- Paraguaçu. Revista Brasileira de Ciências Agrárias, Recife, v. 4, n. 4, p. 440-448, 2009. http://dx.doi.org/10.5039/agraria.v4i4a12

LARCHER, W. Ecofisiologia vegetal. Tradução de Augusto C. Franco. São Carlos: RIMA, 2000. 530 p.

RODRIGUES, L. N. et al. Taxas de crescimento em altura da mamoneira submetida ao estresse hídrico-salino In: CONGRESSO BRASILEIRO DE MAMONA, 3., 2008, Salvador. Anais..., Salvador: SEAGRI: Embrapa Algodão, 2008. 1 CD ROM. p. 84. 
RODRIGUES, L. N. et al. Crescimento e produção de bagas de mamoneira irrigada com água residuária doméstica. Revista Brasileira de Engenharia Agrícola e Ambiental, Campina Grande, v. 13, p. 825-835, 2009.

SANTOS, V. M. et al. Avaliação do crescimento e da produtividade da mamoneira BRS 149, Nordestina em dois níveis tecnológicos. Journal of Biotecnology and Biodiversity, Gurupi, v. 2, n. 3, p. 58-66, 2011.

SEVERINO, L. S. et al. Método para determinação da área foliar da mamoneira. Revista Brasileira de Oleaginosas e Fibrosas, Campina Grande, v. 8, n. 1, p. 753-762, 2004.

SEVERINO, L. S. et al. Otimização do espaçamento de plantio para a mamoneira cultivar BRS Nordestina. Revista Brasileira de Oleaginosas e Fibrosas, Campina Grande, v. 10, n. 1-2, p. 933-999, 2006a.
SEVERINO, L. S. et al. Crescimento e produtividade da mamoneira adubada com macronutrientes e micronutrientes. Pesquisa Agropecuária Brasileira, Brasília, v. 41, n. 4, p. 563-568, 2006 b. http://dx.doi.org/10.1590/S0100-204X2006000400003

SILVA, S. M. S. et al. Dotações hídricas em populações de plantas na cultura da mamoneira cv. BRS Energia. Revista Brasileira de Ciências Agrárias, Recife, v. 4, n. 3, p. 338-348, 2009. http:// dx.doi.org/10.5039/agraria.v4i3a17

SOUZA, A. S. et al. Épocas de plantio e manejo da irrigação para a mamoneira. I - Componentes de produção. Revista Ciência Agrononômica, Fortaleza, v. 38, n. 4, p. 414-421, 2007a.

SOUZA, A. S. et al. Épocas de plantio e manejo da irrigação para a mamoneira. II - Crescimento e produtividade. Revista Ciência Agronômica, Fortaleza, v. 38, n. 4, p. 422-429, 2007 b.

Recebido: 08 abr. 2013 Aprovado: 29 jan. 2015 